\title{
Cognitive Repeaters for Flexible Mobile Data Traffic Offloading
}

\author{
Dennis Wieruch*, Thomas Wirth*, Oliver Braz ${ }^{\dagger}$, Alfons Dußmann ${ }^{\dagger}$, Markus Mederle $^{\dagger}$ and Marc Müller ${ }^{\dagger}$ \\ *Fraunhofer Heinrich Hertz Institute, Berlin, Germany \\ ${ }^{\dagger}$ Commscope, Buchdorf, Germany \\ \{dennis.wieruch, thomas.wirth\}@hhi.fraunhofer.de \\ \{oliver.braz, alfons.dussmann, markus.mederle, marc.mueller\}@commscope.com
}

\begin{abstract}
We investigate the performance of cognitive amplifyand-forward (AF) repeaters in a dense urban environment. The benefit of repeaters is twofold. First, repeaters can compensate penetration losses and serve as wireless backhauling technology to bring high data rates into shielded environments. Second, a cognitive repeater can utilize very flexible different frequency bands and adopt its wireless link to the current frequency reuse plan. In this paper, we propose a new cognitive repeater concept which utilizes multiband signal offloading. Therefore, a cognitive repeater is implemented as a real-time hardware prototype in an amplify-and-forward (AF) relay and deployed in a real LTE-A testbed. The system performance is evaluated by various measurement trials within shielded indoor hotspot environments. It is shown, that cognitive repeaters can take advantage of the signal shielding to the outside and beneficially improve system performance for indoor user hotspots.

Index Terms - amplify-and-forward (AF) relaying, LTE-A field trials, indoor coverage, real-time implementation, measurements, cognitive repeaters
\end{abstract}

\section{INTRODUCTION}

The key challenge in future wireless networks is to find solutions for the enormous increase in data traffic demand as predicted by market research reports [1]. Furthermore, studies show that the majority of data traffic in cellular networks originates or terminates inside of buildings or other shielded environments [2]. Although emerging cellular networks based on 3GPP's long term evolution (LTE) enable high rate access on the air interface, distributing capacity to the actual hotspot areas with high user concentrations remains challenging.

Moreover, the mobile communication industry is suffering from insufficient wireless transport capacity due to limited availability of spectrum. The best part of the spectrum below $1 \mathrm{GHz}$ is very ineffectively used by TV broadcasting services [3], [4]. Recently, ITU has initiated changes [5] similar to the US band allocation, to free up more spectrum below $800 \mathrm{MHz}$. Facing a lot of difficulties implementing the first digital dividend [6] and taking into account the inhomogeneous homologation procedures, especially in Europe, it is questionable how fast this plan can be realized.

On the other hand in various studies it was shown that a shared spectrum usage between primary, e.g. TV broadcasting, and secondary, e.g. cognitive mobile, services is possible [4], [7]. For interference reasons, high-power high-tower TV crossboarder coverage planning usually has to rely on large spectral separation. When plotting a geographic map of TV coverage and using colors to denote received signal strength, areas where the spectrum is unused are left uncolored and therefore appear white. By scanning the frequency spectrum, these often termed white spaces can be used for low power mobile applications [3]. To improve the confidence of local white spaces and overcome challenges like the hidden node problem, the usage of a common geolocation data base for coordination of shared bands is expected to become mandatory. A mobile handheld device or smartphone, for power consumption and space limitation reasons, might have difficulties to cope with multiband support, spectrum scanning and data base connection features. A repeater located at the outside-to-inside transition of a building and plugged to the power network does not have these limitations and could take over these tasks.

Furthermore, outdoor multiple-input multiple-output (MIMO) installations often suffer from high inter-stream correlations. This is especially the case if more than two transmit and receive antennas are used and crosspolarization multiplexing cannot be further exploited since both polarizations are already used. Here, inter-stream interference drastically limits the achievable data rates [8]. On the other hand, rich scattering in indoor environments is perfect for exploiting MIMO benefits [9]. A promising solution to bring the MIMO performance into the shielded environment is the installation of a smart MIMO repeater which can offload data streams to different frequency bands for the relay link and multiplex MIMO streams to a different frequency for the access link. Thus, cognitive repeaters can bring in a very flexible way high data rates to shielded hotspot users.

\section{Cognitive Repeaters}

\section{A. Repeater Solutions}

Repeater solutions can be divided in two main categories: amplify-and-forward (AF) and decode-and-forward (DF) types. While the AF approach amplifies and filters the RF signal, including an optional frequency shift, the DF performs a full de- and re-encoding. The DF repeater allows to remove the overlaid noise by the demapping and decoding process, which improves the signal-to-noise ratio (SNR) of the signal. The price which has to be paid is additional latency which usually makes a complete new re-framing inevitable. In addition, DF requires additional features, e.g. cell ID handling 
or TDD-FDD conversion modules, which add considerable complexity to repeater systems in terms of real-time signal processing and thus results in increased costs. Therefore, in this paper we focus on a AF relaying implementation. Note, the delay of the AF approach depends on the used filters and is $<200 \mathrm{~ns}$ in the used AF repeater hardware. The delay in a DF system is considerable higher in the order of several milliseconds.

\section{B. Cognitive Repeater Concept}

The basic schematic of the cognitive repeater concept is shown in Fig. 1. The cognitive repeater consist of two entities: the donor repeater and the coverage repeater. In the following, we will focus on the downlink (DL) of a MIMO-OFDM based system like 3GPP's LTE. The carrier frequency of the transmitted signals is denoted as $f$. Furthermore, the set $\Gamma$ is introduced, which represents all supported carrier frequencies by the cognitive repeater system. The base station (eNB) transmits on $f_{k}$, whereas the donor repeater is able to transmit on a set of frequencies $\Gamma$. The donor repeater has two amplifyand-forward (AF) strategies: $(i)$ if $f_{b}=f_{k}$ with $f_{b} \in \Gamma$, then pure amplification of the signal received on $f_{k}$ is performed, and $(i i)$ if $f_{b} \neq f_{k}$ with $f_{b} \in \Gamma$, then the received signal is converted from frequency $f_{k}$ to $f_{b}$ and amplified. The coverage repeater is transmitting on carrier frequency $f_{i}$ to serve the indoor terminals. In case where $f_{i} \neq f_{k}$ the users in the indoor and the outdoor environment are served on different carrier frequencies. In the later case, the indoor users are operating in a sub-system independent from the outdoor environment. In case $f_{i}=f_{k}$, the indoor and outdoor signals may interfere with each other. However, signals from the outside are attenuated by the penetration loss of the shielded walls and windows of the building.

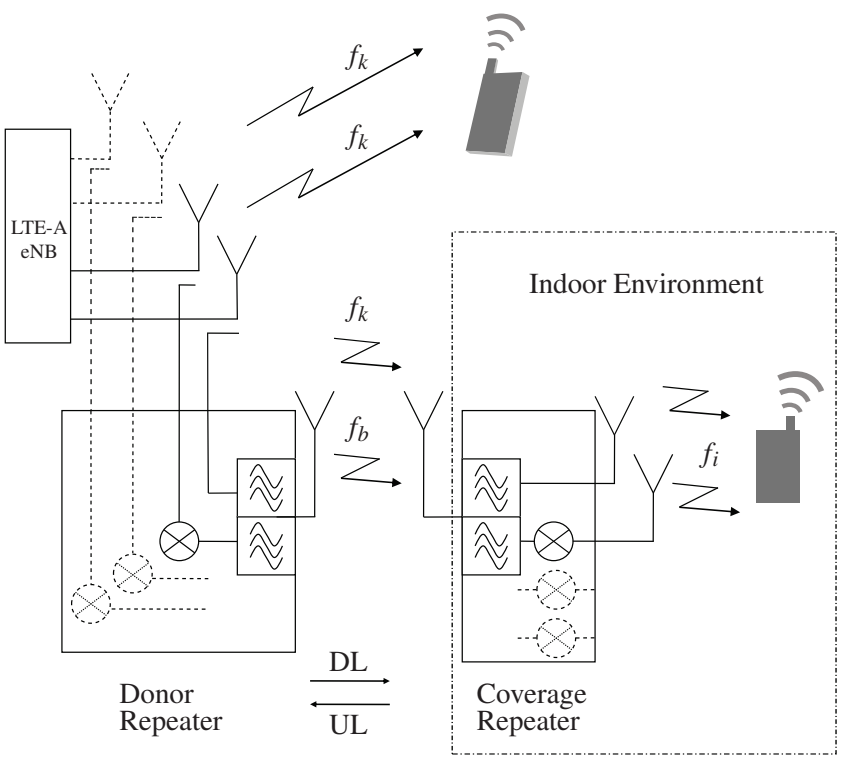

Fig. 1. Schematic of the cognitive repeater concept

In case the repeater cannot exploit the MIMO gain efficiently because of poor properties in the outdoor environment, the streams can be offloaded into different frequency bands. Furthermore, by using its sensing capabilities the cognitive repeater is able to identify white spaces in its supported set of frequencies $\Gamma$. Thus, depending on the requirements of the indoor users and the available white spaces, all streams or just a part of stream can be offloaded into different frequency bands. On the one hand, the performance increases by avoiding inter-stream interference. On the other hand, the signals benefit from isolation between relay link and access link.

\section{Cognitive Pilot Channel}

Knowing the exact location of the repeater system and available cellular access network infrastructure in combination with spectrum scanning capabilities allows cognitive repeaters to identify available unused spectrum. Cognitive repeaters can act as distributed senors and feed this information into a centralized database system. This spectrum usage information can either be transmitted via a wired connection or via a standard wireless interface using a cognitive pilot channel [10].

\section{Use Cases}

1) Fixed Installations: Applications for cognitive repeater deployments are manifold. Especially for temporary deployments with large capacity requirements, e.g. conference events, cognitive repeater installations can be a cost effective solution and work efficient in co-existence with already operated cellular networks, e.g. LTE deployments.

2) Mobile Deployments: Cognitive repeaters can be installed in moving vehicles, e.g. high speed trains. Donor repeaters installed along railway tracks feed the mobile cognitive repeater on local available white spaces. The available white spaces are obtained via a centralized database or local sensing operations and are distributed via the cognitive pilot channel to the cognitive repeater. Furthermore, the maximum allowed transmit power of the coverage repeater can be adjusted automatically to avoid interference by its secondary spectrum usage.

3) Multi-Band Filter Bank Design: Facing constantly shrinking form factor requirements for mobile wireless handsets and being aware that each additional band implemented might result in larger handset dimensions reveals another benefit of the proposed repeater solution. While digital baseband processing can flexibly be realized, the main bottleneck for the introduction of software-defined radio (SDR) techniques [11] remains in the availability of universal programmable RF components. With respect to efficient bandwidth utilization, it is most important to control adjacent channel interference issues. Even though significant progress has been made in recent years in the design of high linear broadband amplifiers, the use of pre- and post-filtering is still indispensable. Bulky filter banks or tuneable low loss filters are easier to be implemented in a repeater system than in a handset.

\section{MeAsurement ScEnARIOS}

To verify the benefits of a cognitive repeater system including wireless backhauling, the test system as shown in 
Fig. 2 was setup at the Heinrich Hertz Institutes Berlin LTE-A Testbed.

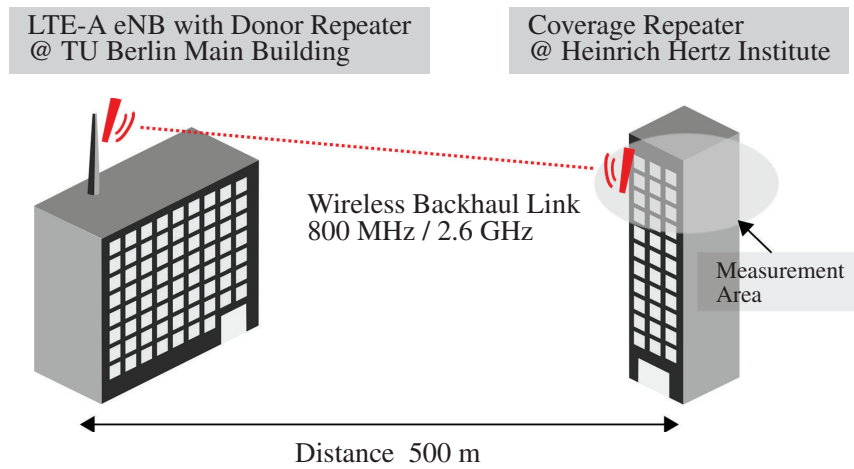

Fig. 2. Schematic drawing of the outdoor backhaul link test setup

The LTE eNB used for the access link implements $2 \times 2$ MIMO and connects to the core network. Furthermore, the eNB is configured to use $20 \mathrm{MHz}$ bandwidth for the LTE downlink (DL), allocated in the digital dividend band at $800 \mathrm{MHz}$. For further description of the parameters of the LTE prototype refer to [9] and [12]. The donor repeater supports MIMO and can be configured to pick up single or multiple signals from the eNB as well as frequency shift streams to the $2.6 \mathrm{GHz}$ UMTS extension band. Thus, it is possible to investigate the performance of several transmission schemes. The coverage repeater, which is located inside of the shielded office building, can pick-up multiple frequency signals and distribute the signals directly or via an optical distribution system. All measurements were performed without additional interference from other cells.

\section{A. Indoor Measurement Setup}

In all performed DL measurements, a single mobile terminal (MT) was used which moved on pre-defined tracks through the office building of the Heinrich Hertz Institute. A typical results when distributing the signals by the coverage repeater via an optical distributed antenna system (DAS) is shown in Fig. 3. For all measurements, the MT was moved along the same tracks at pedestrian speeds. The colors indicated the overall data throughput along the measurement tracks on the 13th floor of the building. The peak data rate of the system under optimal conditions is approx. $150 \mathrm{Mbps}$ for a $2 \times 2$ MIMO configuration. Previous measurement trials with a similar system on different floors of the same building were conducted in [9]. The results showed that an optimal distribution of MIMO system with the coverage repeater can be achieved.

Without DAS system, previous measurement results in [9] showed a dramatic decrease of data rates when moving through the building with outage in approx. $40 \%$ of the cases. This is due to the building construction of this typical office building. Especially new windows with metal protection layers for UV shielding easily cause a signal reduction of $20-30 \mathrm{dBm}$. Furthermore, antenna downtilting of the Macro base stations in the surrounding area leads to low SNR on the higher floors of the building.

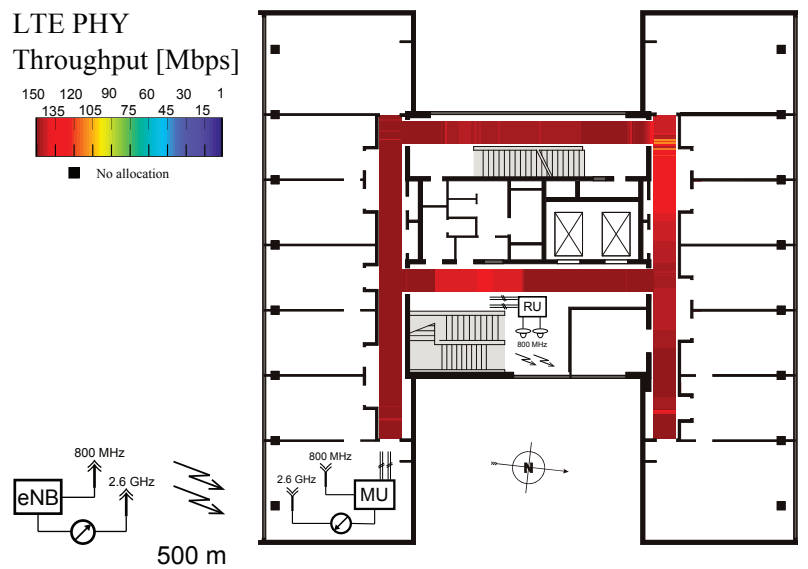

Fig. 3. In-building coverage with cognitive repeater deployment

\section{Measurement Results}

Fig. 5 and Fig. 7 show the physical layer (PHY) throughput distribution curves determined for the data throughput measured in megabits per second for different antenna and system configurations.

As mentioned in the previous section, the peak data rate for this 2x2 LTE-Advanced (LTE-A) MIMO prototype is 150 Mbps. When the number of antennas is doubled to a $4 \times 4$ MIMO system and all antennas are optimally decoupled, the data rates increases linearly with the number of transmit and receive antennas to $300 \mathrm{Mbps}$.

\section{A. Carrier Frequency Offset}

First, measurements were done in order to determine the maximum allowed carrier frequency offset (CFO) within a $2 \times 2$ MIMO repeater system. This measurement determines the required frequency synchronization between the donor and coverage repeater. In this measurement setup, one transmission signal is offloaded at the donor repeater from $800 \mathrm{MHz}$ to $2.6 \mathrm{GHz}$, the second signal is transmitted at the original frequency at $800 \mathrm{MHz}$. A conversion error is manually introduced at the coverage repeater by adjusting the local oscillator frequency. Thus, the $2.6 \mathrm{GHz}$ signal is converted to $800 \mathrm{MHz}$ with a CFO towards the original $800 \mathrm{MHz}$ signal.

The constant offset of $50 \mathrm{~Hz}$ was added stepwise at the coverage repeater. The $\mathrm{CFO}$ was increased until synchronization between eNB and user equipment (UE) was lost, which was at approx. $680 \mathrm{~Hz}$ at the user terminal. The result of this measurement are shown in Fig. 4. The data rate drops from approx. $150 \mathrm{Mbps}$ to $65 \mathrm{Mbps}$ with the maximum possible CFO, see Fig. 4 (right). With a CFO above $700 \mathrm{~Hz}$, the UE fails to synchronize to the eNB. 


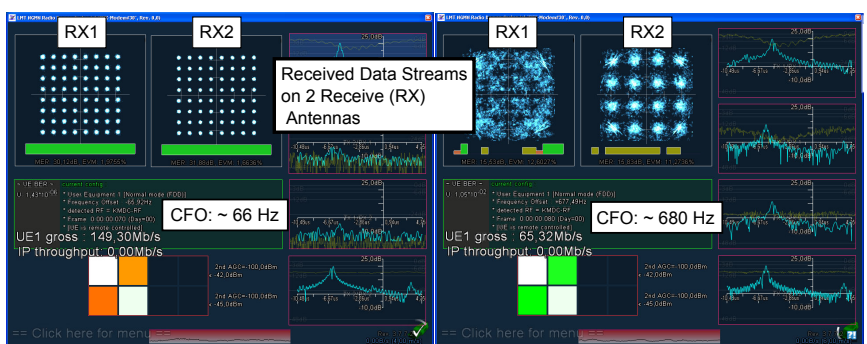

Fig. 4. Signal quality at the measurement UE: low CFO (left), high CFO (right)

\section{B. Frequency Conversion for $2 \times 2$ MIMO}

Several measurement trials were performed for the 2x2 MIMO system with and without frequency conversion. The results in Fig. 5 and curves (a), (b), (c), and (d), show the advantage of the frequency conversion for the $2 \times 2$ MIMO system. The original MIMO signals was in all cases transmitted at $800 \mathrm{MHz}$ from the LTE-A eNB at the TU Berlin main building.

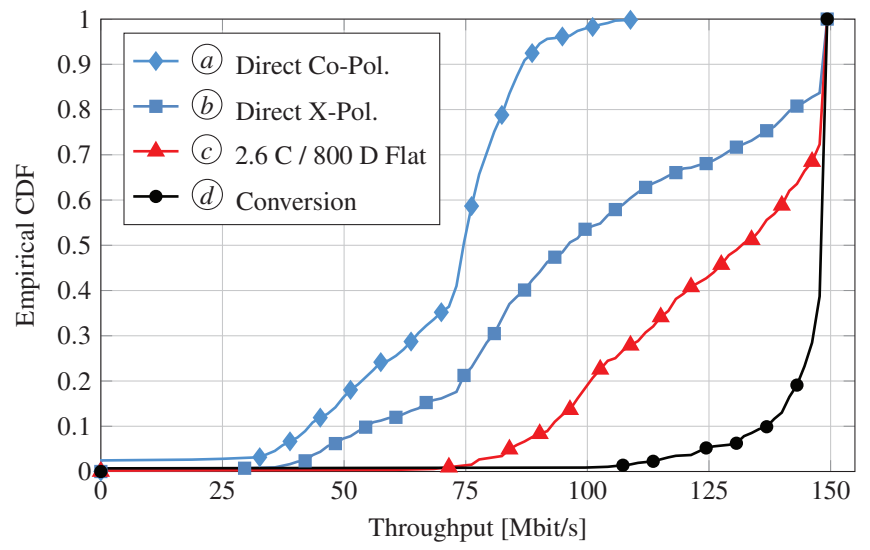

Fig. 5. Rate distribution for different antenna setups

1) Direct Transmission with Co-Polarized Antennas: The first two measurements were done for reference purposes and performed without conversion system. Thus, the direct successfully decoded MIMO data symbols at the MT as transmitted by the eNB were measured.

Fig. 5 (a) shows the throughput distribution if two copolarized antennas are placed with an antenna spacing of $2 \lambda$ at the LTE-A eNB. The identical polarization and the small antenna spacing of the transmit antennas lead to a very small angular spread and thus to a strong correlation between the two transmit streams. As a result, the data streams can no longer be decoded by a MIMO terminal in the shielded environment. The data rate as measured at the MT is thus halved and is below 75 Mbps in more than 50\% of the cases. The highest data rates of $150 \mathrm{Mbps}$ cannot be achieved with this configuration. The data rates remains below $87 \mathrm{Mbps}$ in $90 \%$ of the cases with approx. $2 \%$ outage along the measurement track.

2) Direct Transmission with X-Polarized Antennas: Curve (b) shows the throughput distribution using two cross- polarized antennas at the eNB. The MIMO signals are transmitted at equal frequencies from the outdoor area to the indoor measurement track. By using different polarization directions at the transmit antenna, both transmitted streams are better decoupled than in the previous case. This reduces the inter-stream interference between both MIMO streams. The received data streams can thus be decoded with higher probability at the user terminal. In general, the average data rate increases and is approx. $96 \mathrm{Mbps}$ in 50\% of the cases, and above $100 \mathrm{Mbps}$ in more than $46 \%$ of the cases.

3) Offloading on $2.6 \mathrm{GHz}$ : The setup for the throughput distribution for Fig. 5 (C) is the conversion setup shown in Fig. 1. Here, the MIMO streams at the donor side are split up into two data streams. One transmit stream is transmitted directly at $800 \mathrm{MHz}$ from the eNB to the MT inside of the building. The second data stream is fed into the donor repeater, converted to $2.6 \mathrm{GHz}$ and transmitted to the coverage repeater inside of the building. Here, the stream is converted to the $800 \mathrm{MHz}$ frequency band and distributed inside of the building. This conversion is transparent for the LTE MT which receives and decodes two data streams at $800 \mathrm{MHz}$. By using different frequencies at the eNB, both signal streams can be transmitted into the building without inter-stream interference. In addition, the data stream converted from $2.6 \mathrm{GHz}$ to $800 \mathrm{MHz}$ can be amplified by the coverage repeater in the multiband transmission system. Another advantage of the repeater system is that so-called pick-up antennas at the coverage repeater side can bring an additional $20 \mathrm{~dB}$ gain. As a result, the achievable data rate at the LTE MT is approx. $132 \mathrm{Mbps}$ in more than $50 \%$ of the cases. The data rate is above $100 \mathrm{Mbps}$ in more than $82 \%$ of the cases.

4) Offloading on $2.6 \mathrm{GHz}$, Amplification of $800 \mathrm{MHz}$ : The setup for the measurement result in Fig. 5 (d) is similar to the conversion setup in the previous case. The difference between (c) and (d) is, that in this case the signal stream in the $800 \mathrm{MHz}$ frequency band is picked-up by the coverage repeater and, if necessary, additionally amplified. Thus, both signals, the converted $2.6 \mathrm{GHz}$ and $800 \mathrm{MHz}$ signal, are transmitted inside the building by the coverage repeater. The two data streams are then received and decoded at the MT. By using different frequencies at the base station, both streams can be transmitted into the building without inter-stream interference. In addition, both data streams are amplified. As a result, the achievable data rate at the terminal is approx. $148 \mathrm{Mbps}$ in more than $50 \%$ of the cases. The data rate is above $100 \mathrm{Mbps}$ in more than $99 \%$ of the cases.

Overall, it can be seen that the data throughput can be doubled by utilizing the cognitive transmission methods of the AF relay node.

5) Received Power: Fig. 6 (a)- (d) shows the received power distribution for the different antenna setups. The mean received power for both direct transmission modes (a) and (b) is approx. $-57 \mathrm{dBm}$. For mode (C), the received power can be increased by approx. $8 \mathrm{dBm}$ to an average receive power of $-49 \mathrm{dbm}$. The offloading mode with amplification of the $800 \mathrm{MHz}$ band gives another gain of $8 \mathrm{dBm}$ and achieves 
an average receive power of approx. $-41 \mathrm{dBm}$. Finally, the receive power is in $90 \%$ of the cases above $-50 \mathrm{dBm}$ for the full conversion case in mode (d).

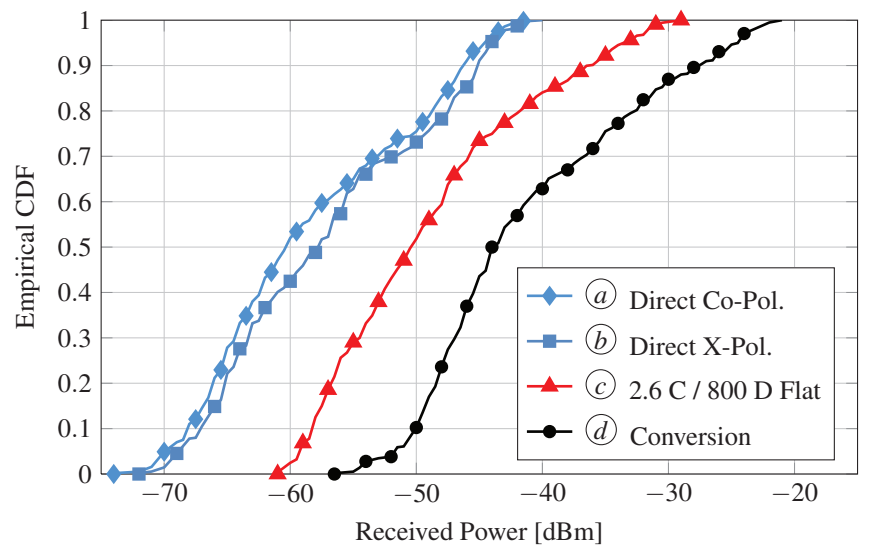

Fig. 6. Received power distribution for different antenna setups

\section{Frequency Conversion for $4 x 4$ MIMO}

Next, Fig. 7 shows the measurement results from Fig. 5 extrapolated to a virtual $4 \times 4$ MIMO system. With optimal decoupling of all data streams, the data rate is scaled linearly with the number of transmit and receive antennas and is in this case doubled to 300 Mbps.

By using cross-polarized antennas for the direct link in the outdoor area, a doubled utilization of the radio channel is achievable even without cognitive methods, also in weakly dispersive environments. The limitation for adequate polarization decoupling is the restricted perpendicular antenna arrangement of the two transmit and receive antennas.

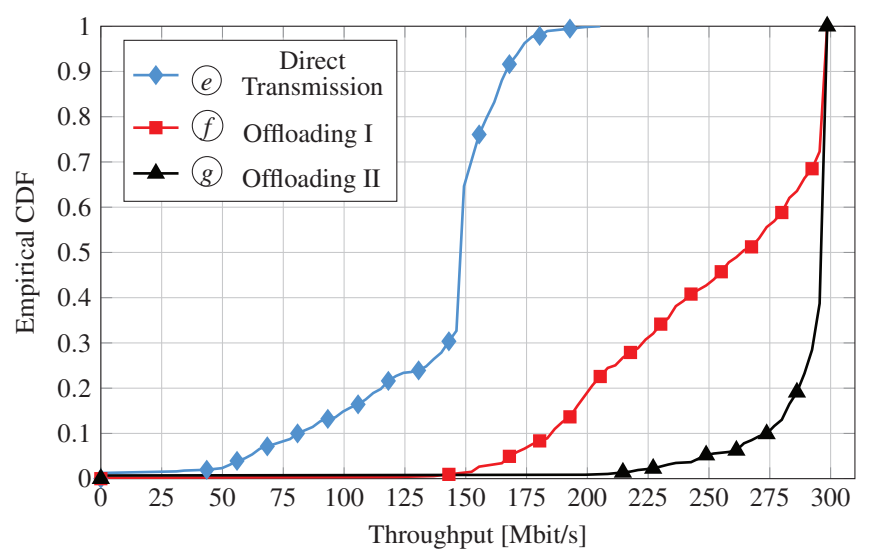

Fig. 7. Cumulative distribution function curves, extrapolated onto a $4 \times 4$ MIMO system.

1) Direct Transmission with X-Polarized Antennas: Fig. 7 (e) shows the measured data rates with the assumption that four transmit streams in the $800 \mathrm{MHz}$ frequency band are transmitted directly into the building. The data streams are received either by a MT with four receive antennas or with two MTs both having two receive antennas. In this context, two dual-X-polarized transmit antennas are used at the eNB. Since in each case the same polarization direction has to be used twice at the eNB, inter-stream interference between both stream will cause inter-stream interference between two data streams and thus performance degradation at the MTs. As a result, the total system throughput degrades to $150 \mathrm{Mbps}$ in $50 \%$ of the cases. In only $30 \%$ of the cases, a higher throughput than $150 \mathrm{Mbps}$ can be achieved, but the distribution remains quite steep. The data throughput remains below $180 \mathrm{Mbps}$ in $98 \%$ of the cases and no doubling of the data rate to $300 \mathrm{Mbps}$ can be achieved at any test point in the measurement scenario.

2) Offloading I and II: In the test scenario for curves $(\oplus$ and (g), the base station transmits four transmit streams via a dual$\mathrm{X}$-polarized antenna configuration as in the previous scenario. However, two different frequency bands are utilized. Two streams are transmitted using $800 \mathrm{MHz}$ and two streams are transmitted using $2.6 \mathrm{GHz}$. The two streams are converted to $2.6 \mathrm{GHz}$ by the donor repeater and converted back to $800 \mathrm{MHz}$ by the coverage repeater to transmit the streams inside the building. By using different frequencies, the transmit streams are now decoupled and there is no inter-stream interference. In addition, the multiband transmission unit within the coverage repeater receives all four data streams. These streams are then amplified and transmitted after frequency reconversion in the $800 \mathrm{MHz}$ frequency band. As a result, the full $300 \mathrm{Mbps}$ can be brought into the building.

According to curve $(1$, a data rate of more than $260 \mathrm{Mbps}$ can thus be achieved in $50 \%$ of the cases, in almost $30 \%$ of the cases it is even possible to achieve the peak data rate of $300 \mathrm{Mbps}$. The result is a large increase in comparison to the first scenario. The gain in throughput is on average $82 \%$.

In curve (g), the transmit power at the coverage repeater was raised by $20 \mathrm{~dB}$ inside of the building. In this case, the data rate of $295 \mathrm{Mbps}$ can be achieved already in more than $50 \%$ of the cases, which corresponds to a gain in throughput of $15 \%$ with respect to curve $(\mathbb{f}$. Overall, this results in an increase of $110 \%$ in comparison with direct transmission in curve (e).

\section{CONClusion}

This paper presented a novel amplify-and-forward (AF) repeater solution which can very effectively be used for mobile traffic offloading in unused frequency bands. The presented concept is a low complexity approach, since no signal processing has to be done within the repeater hardware. It is also very flexible in terms of configuration and therefore enables effective data rate distribution to combat the explosive data rate demand, especially in indoor environments with high user concentration.

While the proof of concept has been performed in prior known frequency ranges only, in the next step frequency agile components, e.g. broadband amplifiers and switchable filter banks, have to be implemented. Relieving mobile handsets from another RF band extension will help to deploy SDR technologies faster as well. Another important task to establish 
will be the connection to a cognitive data base. Especially when talking about isolated mobile coverage cells, e.g. in-train coverage, the dynamic exchange of spectrum information has to be utilized.

\section{ACKNOWLEDGMENT}

This work was funded by the Federal Ministry of Education and Research (BMBF) of Germany in the framework of the Cognitive Mobile Radio (CoMoRa) project under support grant 16BU1200 and 16BU1203.

\section{REFERENCES}

[1] Cisco, "Cisco visual networking index: Global mobile data traffic forecast update, 2011-2016." Cisco, Tech. Rep., 2012, White Paper.

[2] ABIresearch, "In-building wireless systems - passive and active DAS, repeaters, picocells, and femtocells," ABIresearch, Tech. Rep., 2009. [Online]. Available: www.abiresearch.com

[3] V. Valenta, R. Marsalek, G. Baudoin, M. Villegas, M. Suarez, and F. Robert, "Survey on spectrum utilization in Europe: Measurements, analyses and observations," in Proceedings of IEEE CrownCom, June 2010, pp. $1-5$.
[4] Ofcom, "Digital dividend: Cognitive access, statement on licence-exempting cognitive devices using interleaved spectrum," Retrieved September 19th, 2012, from http://stakeholders.ofcom.org.uk/consultations/cognitive/statement/, Ofcom, Tech. Rep., July 2009.

[5] ITU, "WR-C12," ITU, Geneva, Tech. Rep., Feb. 2012, Press Release.

[6] Ofcom, "Second consultation on coexistence of new services in the 800 MHz band with digital terrestrial television," Ofcom, Tech. Rep., Feb. 2012.

[7] J. van de Beek, J. Riihijarvi, A. Achtzehn, and P. Mähönen, "UHF white space in europe - a quantitative study into the potential of the 470-790 MHz band," in Proceedings of IEEE DySPAN, May 2011, pp. 1 -9.

[8] K. Werner, J. Furuskog, M. Riback, and B. Hagerman, "Antenna Configurations for 4x4 MIMO in LTE - Field Measurements," in Proceedings of IEEE VTC 2010-Spring, 2010, pp. 1-5.

[9] T. Wirth, L. Thiele, T. Haustein, O. Braz, and J. Stefanik, "LTE amplify and forward relaying for indoor coverage extension," in Proceedings of IEEE VTC 2010-Fall, Sept. 2010, pp. 1 -5.

[10] J. Perez-Romero, O. Sallent, R. Agusti, and L. Giupponi, "A Novel On-Demand Cognitive Pilot Channel Enabling Dynamic Spectrum Allocation," in Proceedings of IEEE DySPAN. IEEE, 2007, pp. 46-54.

[11] A. Abidi, "The path to the software-defined radio receiver," Solid-State Circuits, IEEE Journal of, vol. 42, no. 5, pp. 954 -966, May 2007.

[12] T. Wirth, V. Jungnickel, A. Forck, S. Wahls, H. Gaebler, T. Haustein, J. Eichinger, D. Monge, E. Schulz, C. Juchems, F. Luhn, and R. Zavrtak, "Realtime multi-user multi-antenna downlink measurements," Proc. IEEE Wireless Communications and Networking Conference (WCNC), Mar. 2008. 